\title{
NOT IN OUR BACKYARD: SPECTROSCOPIC SUPPORT FOR THE CLASH $z=11$ CANDIDATE MACS 0647-JD
}

\author{
Nor Pirzkal ${ }^{1}$, Dan Coe ${ }^{1}$, Brenda L. Frye ${ }^{2}$, Gabriel Brammer ${ }^{1}$, John Moustakas ${ }^{3}$, Barry Rothberg ${ }^{4,5}$, \\ Thomas J. Broadhurst ${ }^{6}$, Rychard Bouwens ${ }^{7}$, Larry Bradley ${ }^{1}$, ArJen van der Wel ${ }^{8}$, Daniel D. Kelson ${ }^{9}$, \\ Megan Donahue ${ }^{10}$, Adi Zitrin ${ }^{11,13}$, Leonidas Moustakas ${ }^{12}$, and Elizabeth Barker ${ }^{1}$ \\ ${ }^{1}$ Space Telescope Science Institute, 3700 San Martin Dr., Baltimore, MD 21218, USA; npirzkal@stsci.edu \\ 2 Steward Observatory/Department of Astronomy, University of Arizona, 933 N Cherry Ave, Tucson, AZ 85721, USA \\ ${ }^{3}$ Department of Physics and Astronomy, Siena College, 515 Loudon Road, Loudonville, NY 12211, USA \\ ${ }^{4}$ Large Binocular Telescope Observatory, University of Arizona, 933 N.Cherry Ave,Tucson AZ 85721, USA \\ ${ }^{5}$ George Mason University, Department of Physics and Astronomy, MS 3F3, 4400 University Drive, Fairfax, VA 22030, USA \\ ${ }^{6}$ Tel Aviv University-Wise Observatory, Israel \\ ${ }^{7}$ Universiteit Leiden, The Netherlands \\ ${ }^{8}$ Max-Planck-Institut fur Astronomie, Heidelberg, Germany \\ ${ }^{9}$ Carnegie Institution of Washington, USA \\ ${ }^{10}$ Dept of Physics and Astronomy, Michigan State University, East Lansing, MI 48824, USA \\ ${ }^{11}$ Cahill Center for Astronomy and Astrophysics, California Institute of Technology, MC 249-17, Pasadena, CA 91125, USA \\ 12 Jet Propulsion Laboratory, M/S 169-506, 4800 Oak Grove Drive, Pasadena, CA 91109, USA \\ Received 2014 November 26; accepted 2015 February 22; published 2015 April 23
}

\begin{abstract}
We report on our first set of spectroscopic Hubble Space Telescope observations of the $z \approx 11$ candidate galaxy, which is strongly lensed by the MACSJ $0647.7+7015$ galaxy cluster. The three lensed images are faint and we show that these early slitless grism observations are of sufficient depth to investigate whether this high-redshift candidate, identified by its strong photometric break at $\approx 1.5 \mu \mathrm{m}$, could possibly be an emission line galaxy at a much lower redshift. While such an interloper would imply the existence of a rather peculiar object, we show here that such strong emission lines would clearly have been detected. Comparing realistic, two-dimensional simulations to these new observations, we would expect the necessary emission lines to be detected at $>5 \sigma$, though we see no evidence for such lines in the dispersed data of any of the three lensed images. We therefore exclude that this object could be a low-redshift emission line interloper, which significantly increases the likelihood of this candidate being a bona fide $z \approx 11$ galaxy.
\end{abstract}

Key words: galaxies: high-redshift - techniques: spectroscopic

\section{INTRODUCTION}

The search for the first galaxies has been at the forefront of extragalactic astronomy ever since the initial discovery that quasi-stellar objects (QSOs) are so distant that their UV spectral features were redshifted into the optical pass-bands (Schmidt 1983). Owing to their high space density, starforming galaxies comprise a large fraction of the high- $z$ galaxy population available to us at the highest redshifts. Gravitational lensing is an efficient method that allows the detection of high redshift star-forming galaxy candidates that would otherwise have luminosities that are too faint to be detected using current space- or ground-based observatories (Wyithe et al. 2011).

A strong $z \approx 11$ candidate was identified by Coe et al. (2013) in observations of the $z=0.591$ galaxy cluster MACSJ $0647.7+7015$. These observations were made as part of the Cluster Lensing And Supernova survey with Hubble (CLASH, Postman et al. 2012) program. Analysis of three separate lensed images (JD1, JD2 and JD3) of the faint red object in nearinfrared pass-bands led Coe et al. (2013) to conclude that this target was a strong candidate for $\mathrm{a} z \approx 11$ galaxy. Three images of the same high redshift candidate were identified with magnification factors estimated to be $\approx 8,7$, and 2 for the JD1, JD2, and JD3 images, respectively. The three images are magnified to F160W AB magnitudes of $\approx 25.9,26.1$, and 27.3, respectively. All three images show no detected flux in

\footnotetext{
${ }^{13}$ Hubble Fellow.
}

observations taken in 15 different Hubble Space Telescope (HST) filters over a wavelength range of 0.2-1.4 $\mu \mathrm{m}$. However, all three were detected in the F140W $(>6 \sigma$ at $1.4 \mu \mathrm{m})$ and F160W ( $>12 \sigma$ at $1.6 \mu \mathrm{m})$ filters. While Coe et al. (2013) ruled out that this source could be a low-redshift interloper by considering a variety of possible low-redshift objects and the strength of the lensing model, their conclusion relied on the detection of this object in only two infrared broadband filters, while this source remained undetected at the longer wavelengths probed by relatively low signal-to-noise Spitzer/IRAC observations at 3.6 and $4.5 \mu \mathrm{m}$. The authors pointed out that the discovery of such an object was consistent with expectations extrapolated from $z \approx 8$ (Bradley et al. 2012), but is in conflict with the significantly lower than expected number of $z>9$ detected by Bouwens et al. (2014) and Oesch et al. (2013). Results from Bradley et al. (2012) and Oesch et al. (2013) were indicative of a dramatic buildup in the number of galaxies and of the cosmic star formation rate density over a very short period of time $(<200 \mathrm{Myr})$ between $z \approx 10$ and 8 . This was later repeatedly confirmed by subsequent observations as well as re-analyses of earlier data (e.g., Bouwens et al. 2014; Ishigaki et al. 2014; Oesch et al. 2014a, 2014b; Zheng et al. 2014). There is currently only one other multiply imaged galaxy at $z \approx 10$ (Zitrin et al. 2014). The only other $z>11$ candidate to date is the $z \approx 12$ candidate UDF12-3954-6284 (Ellis et al. 2013). This source has only been detected in a single band and it can therefore be more easily explained away as being a lower-redshift interloper with strong nebular 
emission (Pirzkal et al. 2013). However, UDF12-3954-6284 remains a possible high-redshift candidate as no emission lines have been conclusively detected in subsequent observations and analyses (Bouwens et al. 2013; Brammer et al. 2013; Capak et al. 2013).

Confirming the nature of high-redshift candidates requires spectroscopic observations. If some of the very few $z>9$ candidates already identified were to be shown to be intermediate redshift sources, then the observed deficit of galaxies at $z \approx 10$ would be even greater, requiring an even more rapid buildup of early galaxies. Unfortunately, spectroscopic confirmation of high-redshift candidates has lagged behind due to the faintness of the sources, the apparent lack of Ly $\alpha$ emission in high-redshift galaxies, and the bright infrared sky background in ground-based observations (Ono et al. 2012; Shibuya et al. 2012). The unlensed Hubble Ultra Deep Field (Beckwith et al. 2006) $z>9$ candidates are significantly fainter $(29.3<\mathrm{F} 160 \mathrm{~W} \mathrm{AB}<29.7)$, prohibiting spectroscopic confirmation with current telescopes and limiting the spectroscopic studies possible with future telescopes. Fortunately, cluster lensing magnifies images of the MACS 0647-JD candidate such that space-based low-resolution spectroscopy is feasible.

While the possibility that this object is a low-redshift emission line galaxy is in contradiction with current lens models, spectroscopically ruling out that this object is not a low-redshift star-forming galaxy is important. There is empirical evidence for the existence of faint moderate redshift galaxies with very strong emission lines. Straughn et al. (2011) identified several extreme emission line objects in Early Release Science observations using the G102 and G141 Wide Field Camera 3 (WFC3) grisms. Their Figure 3, for example, shows the observed spectrum of an F098M 26.87 AB magnitude source (ID 397) at $z=1.76$, which has a very strong $[$ OІІІ $] / \mathrm{H} \beta$ doublet $\left(9.69 \pm 1.310^{-17} \mathrm{erg} \mathrm{s}^{-1} \mathrm{~cm}^{-2}\right.$, observed equivalent width $(\mathrm{EW}) \approx 5000 \AA)$. This emission line is sufficient to produce a photometric break of nearly two $\mathrm{AB}$ magnitudes and is nearly as extreme as what would be required to reproduce the flux measured in MACS 0647-JD $\left(\approx 5 \times 10^{-17} \mathrm{erg} \mathrm{s}^{-1} \mathrm{~cm}^{-2}\right)$. See also Frye et al. (2012) for other examples of extreme emission line galaxies with large single emission line fluxes of up to $1.38 \times 10^{-16} \mathrm{erg} \mathrm{s}^{-1} \mathrm{~cm}^{-2}$.

Redshift confirmation is therefore essential to definitively rule out a low-redshift emission line interloper (or perhaps discover the true nature of a very interesting galaxy).

\section{OBSERVATIONS}

This paper is based on a set of new $H S T$ near-infrared stilless spectroscopic observations of the MACSJ 0647.7+7015 cluster. As part of a follow-up project, we will be observing this cluster for 12 orbits using the G141 grism on the WFC3. These observations are split in three epochs, revisiting this field at different position angles on the sky. The position angles were chosen to minimize the amount of spectral contamination in the spectra of our target and to provide stronger, independent detections of any spectral break or emission lines in the spectra of this source. We have obtained our first set of observations, which we present here. The data were processed using the aXe data reduction package (Pirzkal et al. 2001; Kümmel et al. 2009), using a custom sky subtraction step that includes different components for Zodiacal light, earth limb, and stray light. More details on this sky subtraction can be found in
Brammer et al. (2014). We used archival data to create mosaics of the field in the F105, F125W, F140W, and F160W filters and to model the spectral energy distribution (SED) of every object in the field. This allowed us to model the spectral contamination caused by nearby objects using aXe's FluxCube quantitative estimates. This approach also allowed us to perform both regular and optimally weighted spectral extractions. Estimating spectral contamination is crucial when analyzing slitless spectroscopic observations because overlapping spectra can cause features that look very similar to spectral breaks and zeroth dispersion orders of nearby sources can also look similar to strong emission lines. A complete understanding of the field is therefore needed to rule out spectral contamination as the cause of any feature we detect in a spectrum.

\section{HIGH-REDSHIFT OR LOW-REDSHIFT EMISSION LINE INTERLOPER?}

While Coe et al. (2013) suggest that the most plausible explanation for the detection of MACS 0647-JD in the F140W and F160W filters is that the object is a lensed galaxy at $z=11$, it is not the only possible scenario. Among the possible scenarios considered by the authors, one consists of the coincidental presence of two emission lines, one in each filter, to mimic a photometric break.

To further explore this, we started by re-fitting the data from Table 2 of Coe et al. (2013) using the Monte Carlo Markov Chain approach (MCMC) described extensively in Pirzkal et al. (2012) and used to analyze other high-redshift candidates (Pirzkal et al. 2013). Our approach uses Bruzual \& Charlot (2003) stellar population models, a Salpeter initial mass function, and SSP models. The free parameters are the redshift, stellar mass, stellar age, extinction level (Calzetti et al. 2000), metallicity, and the ionizing photon escape fraction. This new analysis confirmed that the Coe et al. (2013) photometry were better fit, especially at bluer wavelengths, by a template consistent with a high-redshift Lyman break object at $z \approx 11$. Figure 1 demonstrates that a $10^{10} M_{\odot}$ galaxy at a redshift of $z \approx 11$ provides a very good fit. Although, as we show, such a model would require a significant amount of flux, about the same level that was detected in the F160W filter, in the IRAC bands at 3.6 and $4.5 \mu \mathrm{m}$, the IRAC data are not very sensitive and are consistent with a high-redshift Lyman-Break galaxy model.

Since the MCMC SED fitting approach allows us to fully explore our input parameter space, we can examine what low-redshift models $(z<2.5)$ best fit the photometric observations. However, this approach is ultimately limited by our handling of the nebular emission process, which we assume here to be the source of the high EW lines in the spectra of our simulated star-forming galaxies. In Figure 2 , we show two distinct cases, one at $z \approx 1.4$ and one at $z \approx 2.2$, where a young (few $10^{6} \mathrm{yr}$ old) star-forming galaxy with strong nebular activity can reproduce the broadband observations. In our treatment of nebular emission, we have assumed the same ionizing photon escape fraction as for the nebular emission. The detailed description of how nebular emission is simulated can be found in Pirzkal et al. (2012). Ultimately, it is the ratio of the nebular line emission to the continuum emission which limits how much of the broadband photometric break can be reproduced by the nebular emission lines of our models. We also must resort to an amount of extinction large enough $\left(A_{v} \approx 3-4\right)$ to quench the 


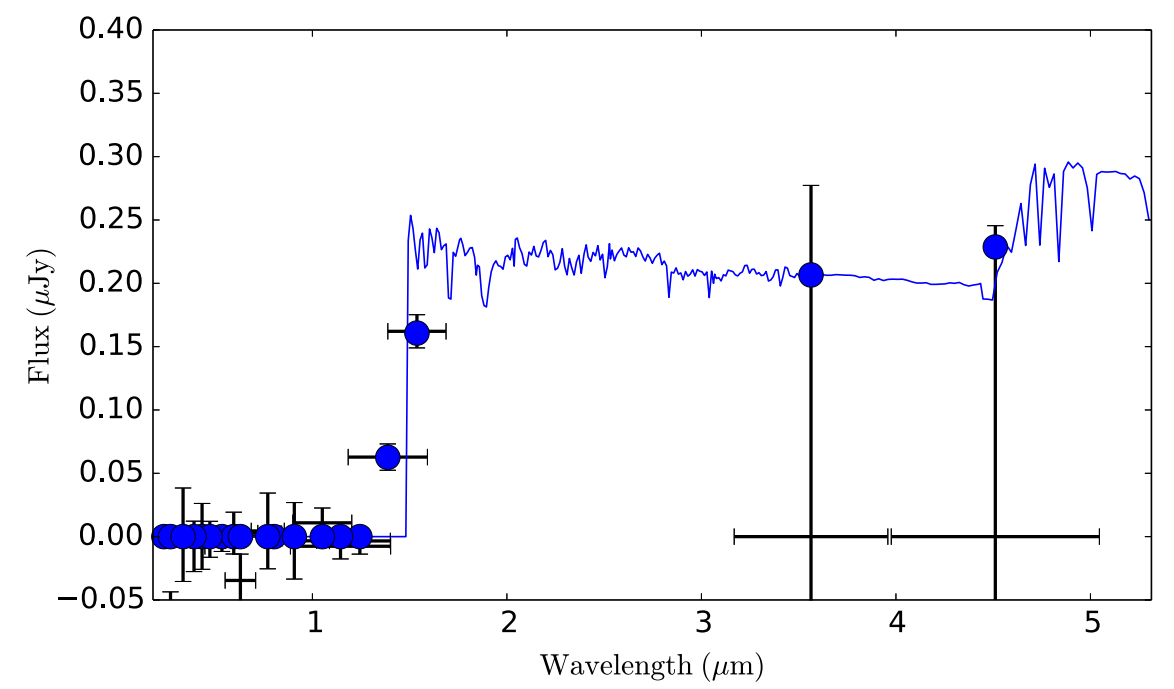

Figure 1. The observed broadband flux levels for JD1 (error bars) and the spectrum of a $z=11.2,10^{10} M_{\odot}$ Lyman Break Galaxy. The model fluxes (blue circles) are in good agreement with the observations.
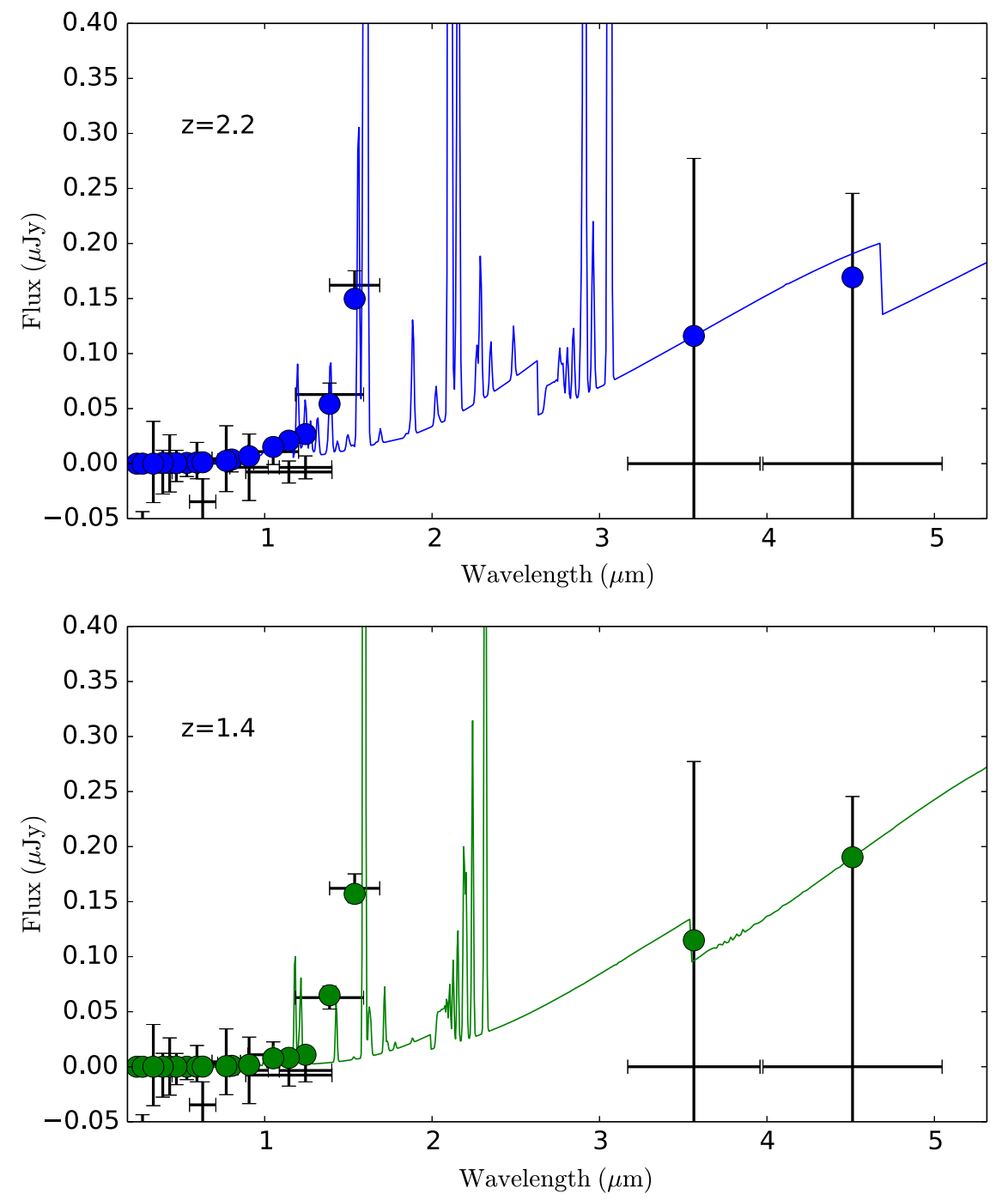

Figure 2. The observed broadband flux levels for JD1 (error bars) and the spectra of two young emission line objects at $z=2.2$ (top panel) and $z=1.4$ (bottom panel). The model fluxes (solid circles) are in good agreement with the observations, although the observed break is sharper than what the models allow when including the nebular continuum emission, as we did here. Models that reproduce the observed break can be generated by allowing a strong $z \approx 1.4 \mathrm{H} \alpha$ or a strong $z \approx 2.2[\mathrm{OIII}]$ emission line to be the dominant source of the light detected using the HST F140W and F160W filters. Several such models at $z \approx 1.4 \pm 0.05$ and $z \approx 2.2 \pm 0.05$ are in principle possible. 


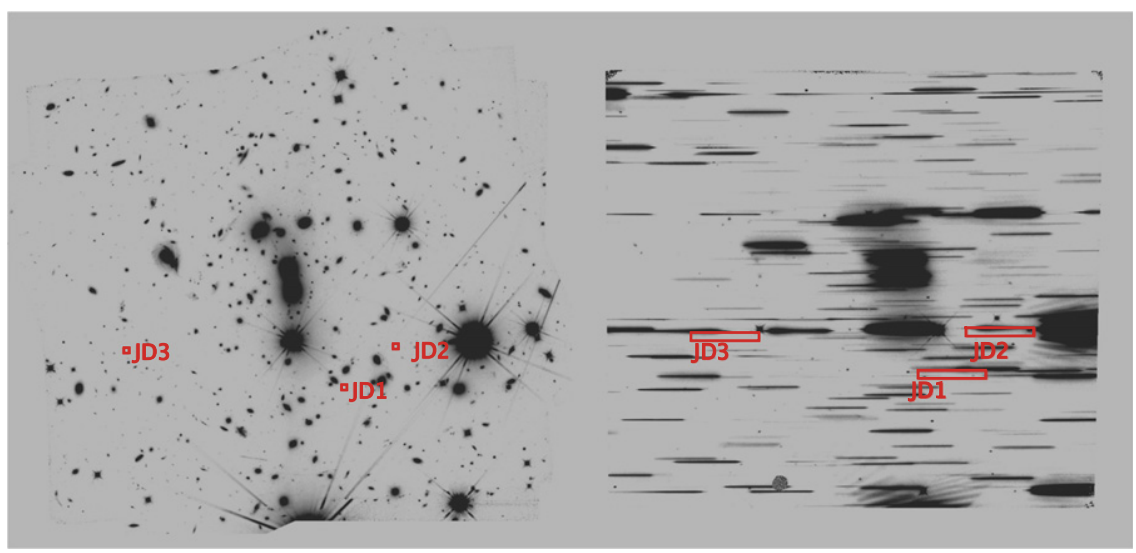

Figure 3. Left panel: composite (F105W+F125W+F140W+F160W) image of CLASH MACSJ 0647. The three lensed images of MACSJ 0647-JD are shown. Right panel: the same field, dispersed using the HST G141 grism. The locations of the JD1, JD2, and JD3 lensed images are shown in the left Panel. The locations of the spectra of JD1, JD2, and JD3 in the G141 data are shown in the right Panel.
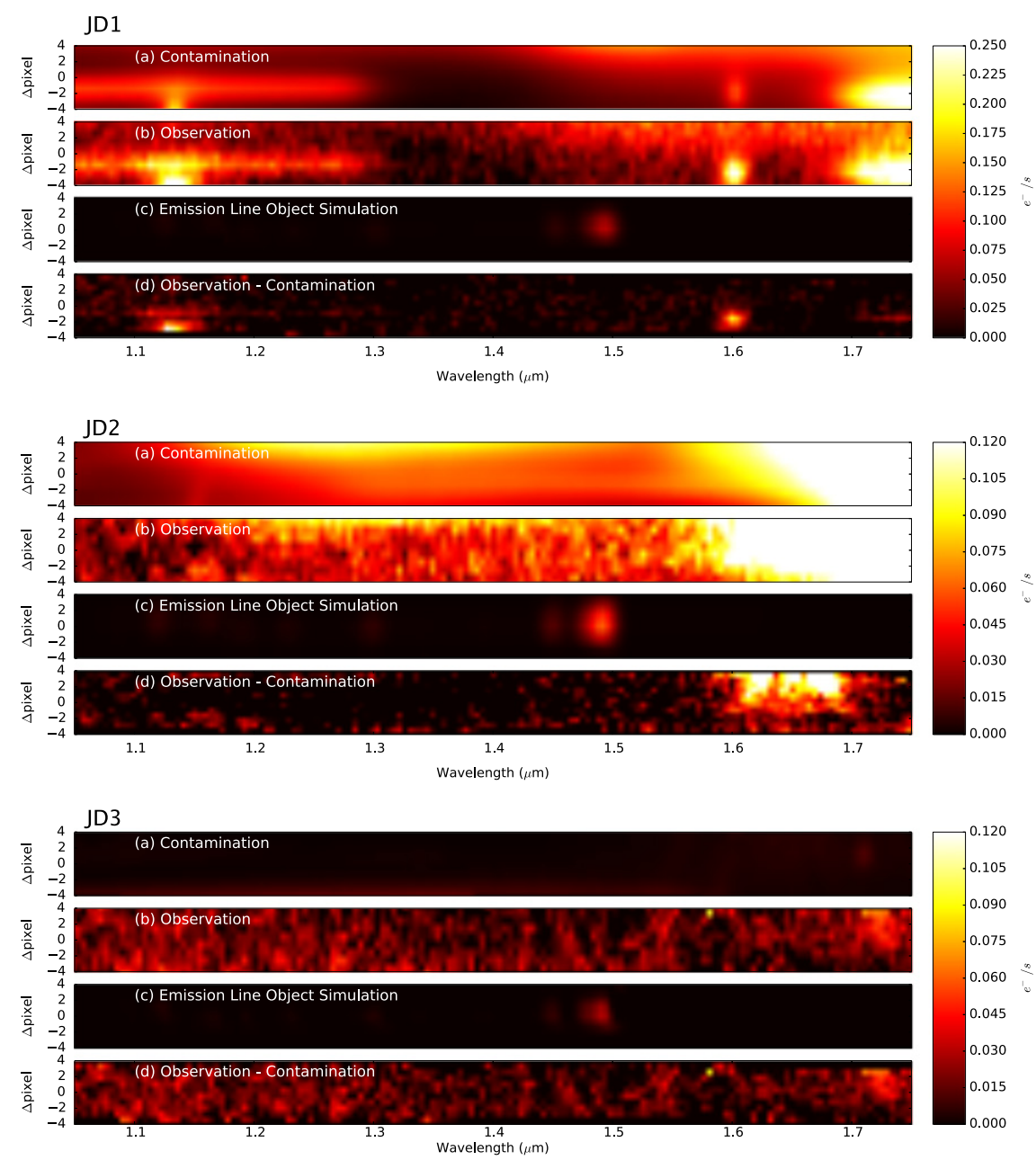

Figure 4. G141 observation of the lensed images JD1, JD2, and JD3. Panels (a) show the model of the spectral contamination from all of the sources in the field. Panels (b) show our observations. Panels (c) show our G141 simulations of what the lensed images would look like dispersed if their spectrum was that of the emission line object shown in Figure 2 . As these simulations of a $z \approx 2.2 \mathrm{H} \beta+[$ OIII] emission line galaxy show, an emission line at $\approx 1.5 \mu \mathrm{m}$ bright enough to reproduce the observed photometric break would be easily detected in our observations of JD1 and JD2. We see no evidence of such a strong emission line in panels (d) where we show our contamination subtracted observations of JD1, JD2, and JD3. The contamination estimate is accurate and subtracts cleanly from the actual observations. The main residual is the two point like zeroth orders at $1.13 \mu \mathrm{m}$ and $1.6 \mu \mathrm{m}$. This is expected, as zeroth orders are relatively poorly calibrated and hence only approximately simulated. These are however located far away, particularly in the $y$-direction, from where we would potentially expect an emission line from JD1. 

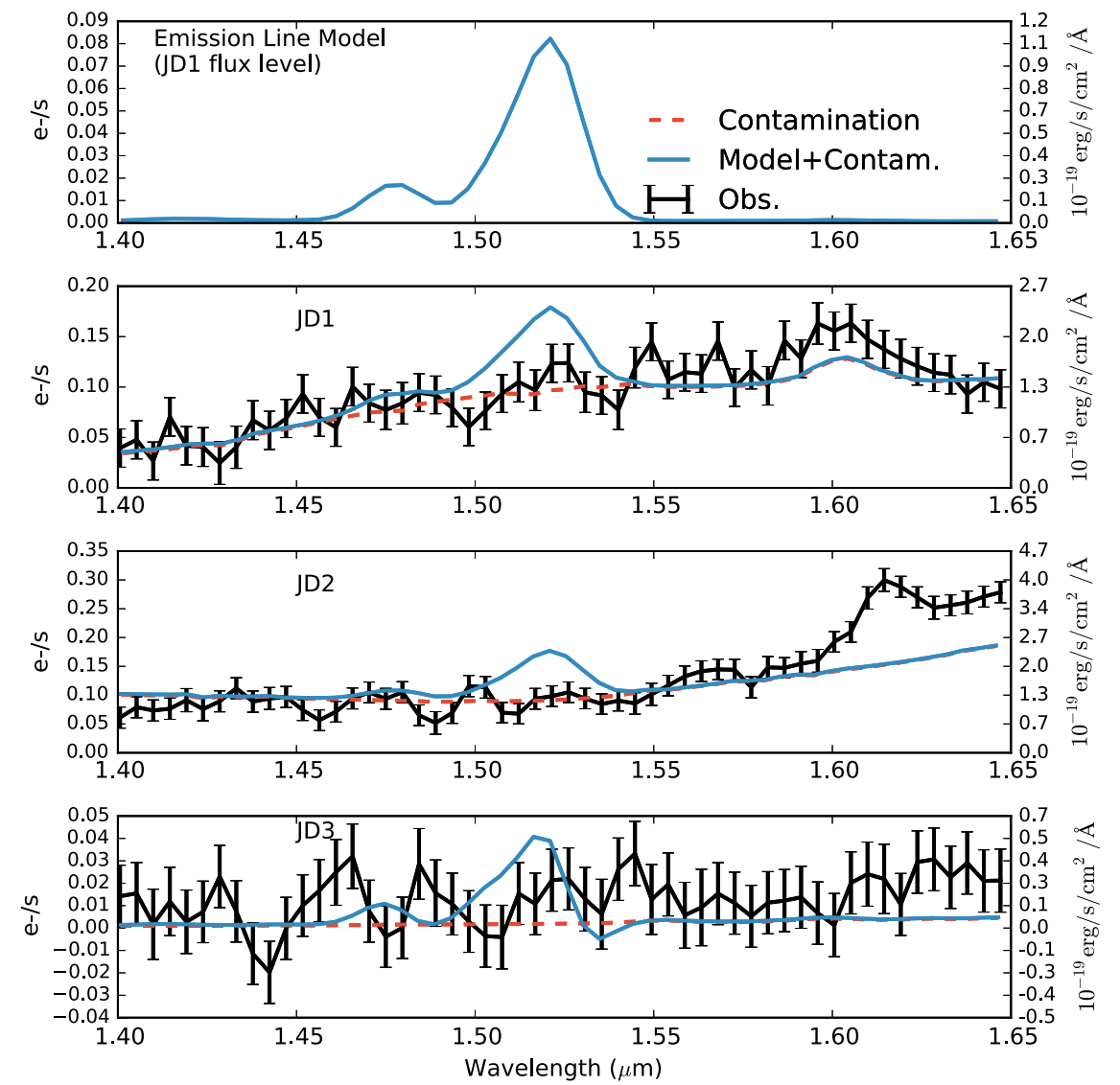

Figure 5. Extracted spectra of JD1, JD2, and JD3 using a narrow extraction window of $\approx 1.5$ pixel to maximize the signal-to-noise (line with error bars). We also show the emission line model spectrum (solid line) of a $z \approx 2.2 \mathrm{H} \beta+[\mathrm{OIII}]$ line interloper in the top panel for JD1. This is the same spectrum shown in Figure 2 . The expected contamination level is shown using a dash line. The contamination is caused by the overlapping spectra of other sources in the field. The emission line models for JD2 and JD3 are similar to the one shown in the top panel for JD1 when accounting for the slight difference in spatial size and lower fluxes.

amount of blue light, including the nebular continuum light, so that such an object can remain undetected in broadband filters blue-ward below $\approx 1.5 \mu \mathrm{m}$. As Figure 2 shows, a $z \approx 2.2$ galaxy with $\mathrm{H} \beta+\left[\mathrm{O}\right.$ III] lines of combined flux of $\approx 5 \times 10^{-17}$ $\mathrm{erg} \mathrm{s}^{-1} \mathrm{~cm}^{-2}$, with a rest-frame EW of $\approx 3000 \AA$, reproduces the observed fluxes, with $[\mathrm{O}$ III] $5007 \AA$ redshifted just redward of F140W, contributing the extra observed flux to F160W. Similarly, a $z \approx 1.4$ galaxy with equally strong $\mathrm{H} \alpha$ emission could also possibly reproduce the observations.

Our multiple nebular emission lines model strongly constrains the redshifts at which we would expect a low-redshift interloper to be $z \approx 1.4 \pm 0.05$ (strong $\mathrm{H} \alpha$ emission) and $z \approx 2.2 \pm 0.05$ (strong $\mathrm{H} \beta+[\mathrm{O}$ III] emission). As shown in Figure 2, in order to produce the observe F140W flux levels, fainter emission lines are required. This places strong constraints on the wavelength range at which strong emission lines are expected. Even if our detailed treatment of nebular emission was inaccurate, the observed photometric break implies the existence of some sort of bright emission line between 1.2 and $1.7 \mu \mathrm{m}$ (the combined wavelength coverage of the F140W and F160W filters). This is well within the bandpass of the G141 observations.

As demonstrated here, it is possible to create a scenario in which emission lines of a relatively low- $z$ galaxy can mimic the photometric break of a higher-redshift object. While these models would have to be cherry-picked and might appear to be unphysical and contrived, the simplest and most direct way to rule out that any of these scenarios are taking place is by demonstrating the absence of strong emission lines in spectroscopic observations of this source.

\section{SLITLESS OBSERVATIONS}

The confirmation of a low-redshift emission line interloper would imply the existence of a rather peculiar galaxy. The HST WFC3 grism mode and its resolution of a few tens of angstroms per resolution element is extremely adept at detecting emission line objects (Straughn et al. 2011) and provides us with an opportunity to test the scenario proposed in the previous section.

As shown in the middle panel of Figure 3, the WFC3 Grism field is extremely crowded and slitless spectroscopic observations are therefore difficult. Most spectra, especially the ones from fainter sources such as the three lensed images of MACS 0647-JD, are contaminated by the spectra of nearby objects. On the other hand, the WFC3 G141 grism is well-understood, well-calibrated, and WFC 3 has has been remarkably stable over the past $6 \mathrm{yr}$. We are therefore able to generate very accurate two-dimensional simulations of what this field would look when observed with the WFC3 G141 grism. The inputs of these simulations are mosaics of the entire MACSJ 0647.7 +7015 field using data from the CLASH program. Mosaics in the F105W, F125W, F140W, and F160W can then be used to detect the footprint and estimate the spectral energy distribution of every object in the field. Simulations are then generated by using the aXeSIM package, along with the photometric data and the latest WFC3 G141 calibration products. With the exception of the still poorly calibrated zeroth spectral order, the 

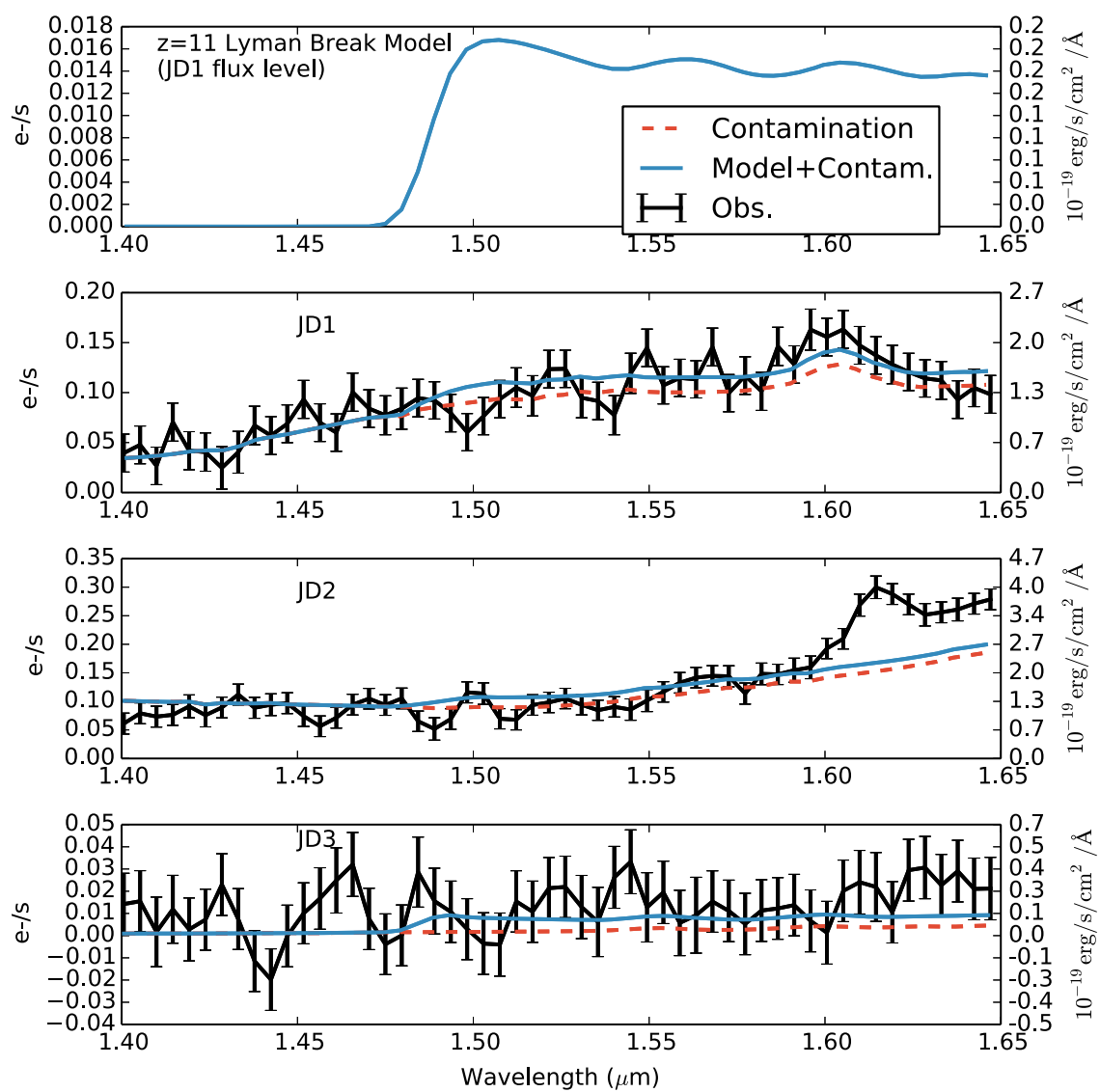

Figure 6. Extracted spectra of JD1, JD2, and JD3 using a narrow extraction window of $\approx 1.5$ pixel to maximize the signal-to-noise (line with error bars). We also show the emission line model spectrum (solid line) of a high-redshfit LBG in the top panel for JD1. This is the same spectrum shown in Figure 2. The expected contamination level is shown using a dash line.

resulting simulations are a very good match to our observations. These simulations are similar to what is done when computing contamination and optimal extraction weigths as part of routine grism extraction (e.g., Pirzkal et al. 2004; Brammer et al. 2012). The observations and the simulations are shown in the middle and right panels of Figure 3, respectively.

Figure 4 qualitatively compares our shallow observations to our simulated dispersed image of the strong emission line source already shown in Figure 2. In these simulations, and since these three lensed images are partially resolved, we have conservatively set the size of the emission line region to be equal to the measured sizes of JD1, JD2, and JD3 in the broadband images. These sizes were measured from the available broadband imaging and identical to the ones listed in Coe et al. (2013). Our simulated emission lines are therefore more spatially diluted than they would be if we had simply assumed an unresolved point source. It is immediately obvious that any emission line(s) bright enough to reproduce the observed broadband photometric break would be detected in three orbit grism observations. As Figure 4 shows, there is no sign of such an emission line in our slitless observations for either JD1, JD2, or JD3, demonstrating with little doubt that the CLASH $z \approx 11$ candidate is devoid of any bright emission lines in the wavelength range covered by the G141 grism. This significantly strengthens the case that this source is a $z \approx 11$ Lyman-break galaxy.

For a more quantitative comparison, we show the extracted one-dimensional spectra of the three MACSJ 0647-JD sources in Figure 5. As this figure shows, the low spectral resolution of the G141 grism would make the emission lines shown in
Figure 2 less prominent and broader. These lines would nevertheless remain readily detectable at the $>5 \sigma$ level. As we described in Section 3, there are actually narrow ranges of redshifts $(\Delta z \approx 0.05)$ over which our models can reproduce the broadband photometric break. While this would result in the emission line in Figure 4 to be shifted, it would remain easily detectable in at least JD1 and JD3, where contamination is either at longer wavelength, or vertically offset from where we would expect an emission line to be located.

Figure 5 shows the spectra in units of $\mathrm{e}^{-} \mathrm{s}^{-1}$ with the corresponding flux limits shown on the right axis. We show these extracted spectra in these units, as their noise level is sky dominated and approximately constant $\left(\approx 0.02 \mathrm{e}^{-} \mathrm{s}^{-1}\right)$. Figure 6 shows the extracted spectra of the three lensed images as well as that of the LBG presented in Figure 1. The expected flux levels for JD1, shown in the top panel of this figure are $\approx 0.02 \mathrm{e}^{-} \mathrm{s}^{-1}$ and the relatively high level of contamination (red dashed line) renders the detection of the continuum impossible in these observations. The forthcoming observations, chosen to avoid contamination, will triple the integration time and greatly improve our odds of detecting the continuum emission from this source.

\section{CONCLUSION}

We have presented results based on our shallow, first epoch slitless spectroscopic observations of the galaxy cluster MACSJ $0647.7+7015$. These data have allowed us to obtain the first spectroscopic observations of the three lensed images 
of the $z \approx 11$ LBG candidate MACSJ 0647-JD of Coe et al. (2013). Following a detailed modeling of the entire field of view, we showed that we can exclude a scenario in which the photometric break at $1.5 \mu \mathrm{m}$ in the SED of this object could be caused by a large EW emission line. Any emission line strong enough to produce the observed broadband photometric break would have been easily detected in these observations. We conclude that this object remains an extremely strong candidate for a genuine $z \approx 11$ galaxy. Based on the simulations in this paper, we expect the continuum level red-ward of the Lyman Break to be detected in planned deeper future observations.

We would like to thank the referee for his or her comments and suggestions to improve the quality of this paper. Support for AZ was provided by NASA through the Hubble Fellowship grant \#HST-HF2-51334.001 A awarded by STScI. This work was supported in part by grant HST-GO-13317.13 from the Space Telescope Science Institute, which is operated by AURA under the NASA contract NAS5-26555.

\section{REFERENCES}

Beckwith, S. V. W., Stiavelli, M., Koekemoer, A. M., et al. 2006, AJ, 132, 1729

Bouwens, R. J., Oesch, P. A., Illingworth, G. D., et al. 2013, ApJL, 765, L16
Bouwens, R. J., Bradley, L., Zitrin, A., et al. 2014, ApJ, 795, 126

Brammer, G. B., van Dokkum, P. G., Franx, M., et al. 2012, ApJS, 200, 13

Brammer, G. B., van Dokkum, P. G., Illingworth, G. D., et al. 2013, ApJL, 765, L2

Brammer, G. B., Pirzkal, N., McCullogh, P., et al. 2014, HST WFC3-ISR, TBD Bradley, L. D., Trenti, M., Oesch, P. A., et al. 2012, ApJ, 760, 108

Bruzual, G., \& Charlot, S. 2003, MNRAS, 344, 1000

Calzetti, D., Armus, L., Bohlin, R. C., et al. 2000, ApJ, 533, 682

Capak, P., Faisst, A., Vieira, J. D., et al. 2013, ApJL, 773, L14

Coe, D., Zitrin, A., Carrasco, M., et al. 2013, ApJ, 762, 32

Ellis, R. S., McLure, R. J., Dunlop, J. S., et al. 2013, ApJL, 763, L7

Frye, B. L., Hurley, M., Bowen, D. V., et al. 2012, ApJ, 754, 17

Ishigaki, M., Kawamata, R., Ouchi, M., et al. 2014, arXiv:1408.6903

Kümmel, M., Walsh, J. R., Pirzkal, N., et al. 2009, PASP, 121, 59

Ono, Y., Ouchi, M., Mobasher, B., et al. 2012, ApJ, 744, 83

Oesch, P. A., Bouwens, R. J., Illingworth, G. D., et al. 2013, ApJ, 773, 75

Oesch, P. A., Bouwens, R. J., Illingworth, G. D., et al. 2014, ApJ, 786, 108

Oesch, P. A., Bouwens, R. J., Illingworth, G. D., et al. 2014, arXiv:1409.1228

Pirzkal, N., Pasquali, A., \& Demleitner, M. 2001, ST-ECF Newslett, 29, 5

Pirzkal, N., Xu, C., Malhotra, S., et al. 2004, ApJS, 154, 501

Pirzkal, N., Rothberg, B., Nilsson, K. K., et al. 2012, ApJ, 748, 122

Pirzkal, N., Rothberg, B., Ryan, R., et al. 2013, ApJ, 775, 11

Postman, M., Coe, D., Benítez, N., et al. 2012, ApJS, 199, 25

Schmidt, M. 1983, Natur, 197, 1040

Shibuya, T., Kashikawa, N., Ota, K., et al. 2012, ApJ, 752, 114

Straughn, A. N., Kuntschner, H., Kümmel, M., et al. 2011, AJ, 141, 14

Wyithe, J. S. B., Yan, H., Windhorst, R. A., \& Mao, S. 2011, Natur, 469, 181

Zheng, W., Shu, X., Moustakas, J., et al. 2014, ApJ, 795, 93

Zitrin, A., Zheng, W., Broadhurst, T., et al. 2014, ApJL, 793, LL12 